\title{
A NEW GENERALIZED EXPONENTIAL DISTRIBUTION: PROPERTIES AND APPLICATIONS
}

\author{
JAWAD HUSSAIN ASHRAF ${ }^{1, *}$, MUNIR AHMAD $^{1}$, A. KHALIQUE ${ }^{1}$, ZAFAR IQBAL $^{2}$ \\ ${ }^{1}$ School of Social Sciences, National College of Business Administration $\&$ Economics Lahore, Pakistan \\ ${ }^{2}$ Department of Statistics, Government Post Graduate College Gujranwala, Pakistan
}

${ }^{*}$ Corresponding author: miyanjawad@gmail.com

\begin{abstract}
The exponential distribution is a popular statistical distribution to study the problems in lifetime and reliability theory. We proposed a new generalized exponential distribution, wherein exponentiated exponential and exponentiated generalized exponential distributions are sub-models of the proposed distribution. We study several important statistical and mathematical properties of the newly developed model and provide the simple expressions for the generating function, moments and mean deviations. Parameters of the proposed distribution are estimated by the technique of maximum likelihood. For two real data sets from the field of biology and engineering, the proposed distribution is compared to some existing distributions. It is found that the proposed model is more suitable and useful to study lifetime data. Thus, it gives us another alternative model for existing models.
\end{abstract}

\section{INTRODUCTION}

Some authors discussed the Gompertz-Verhulst distribution whose distribution is defined by

$$
G(x)=\left(1-\xi \mathrm{e}^{-\vartheta x}\right)^{\alpha}, \quad x>\frac{1}{\vartheta} \ln \xi .
$$

For $\xi=1$, the exponentiated exponential (EE) or generalized exponential (GE) distribution by [5] is submodel of Eq.(1.1). They developed the exponentiated-G family for any parent distribution by $G(x)=H(x)^{\delta}$,

Received May $26^{\text {th }}, 2020$; accepted June $23^{\text {rd }}$, 2020; published July $17^{\text {th }}, 2020$.

2010 Mathematics Subject Classification. 60E05, 62N05, 62F10, 62P10, 62P25, 62P30.

Key words and phrases. exponential distribution; exponentiated type distributions; generalized exponential distribution; lifetime distributions; moments; maximum likelihood estimation.

(C)2020 Authors retain the copyrights of their papers, and all open access articles are distributed under the terms of the Creative Commons Attribution License. 
where $H(x)$ is an arbitrary cumulative distribution function (cdf) and $\delta$ is a positive real number. It is also called Lehmann type I alternative. Various authors developed different exponentiated distributions for various standard distributions and studied their properties. [25] provided a list of thirty exponentiated type distributions. The EE distribution is comprehensively studied in different papers by [6], [21], [7,8], and [22,23] is defined for $(x>0)$ by

$$
G(x)=\left(1-\mathrm{e}^{-\vartheta x}\right)^{\alpha} .
$$

For $\alpha=1$, the special case of Eq.(1.2) is the exponential distribution. The EE distribution is effectively used for studying the skewed data. $[9,10]$ and [13] presented important works on comparison of the EE distribution with some will-known close distributions like the gamma, log-normal and Weibull distributions. [11] further used the information to compare the EE and Weibull distributions because both of them provide very close fits.

[12] revisted some existing properties of the EE model. The beta exponential (BE) distribution is developed by using the Beta-G family by [18], where the EE distribution is a particular case of the BE distribution. [1] generalized the EE distribution and developed the beta generalized exponential (BGE) distribution, wherein the BE and EE distributions are particular cases of the BGE distribution. [17] reviewed the several important characteristics of the EE distribution and derived simple explicit expressions for some properties. [24] generalized the exponential distribution by the Marshall-Olkin family.

[19] generalized the gamma and Weibull distributions in similar way the EE distribution [16]. However, they used another type of exponentiated distribution $G(x)=1-[1-H(x)]^{\beta}$ to generalize the Gumbel and Fréchet distributions. It is also called Lehmann type II alternative. [3] introduced the exponentiated generalized (EG) family as $G(x)=\left[1-\{1-H(x)\}^{\gamma}\right]^{\delta}$, where $\gamma>0$ and $\delta>0$ are additional shape parameters. This class is an extension of both exponentiated type distributions. In recent past, several authors used the EG family for generalizing the distributions.

The rest of the paper is organized as follows: Section 2 introduce the new generalized exponential distribution and discuss some important aspects of the proposed distribution. Section 3 extensively studies the basic properties of the proposed distribution. Section 4 deals with linear presentation of the distribution. In Section 5, We derive the mathematical properties by employing the linear representation of the proposed distribution given in the previous section. Section 6 studies the maximum likelihood estimation and provides the application of the proposed model to real data sets. Finally, concluding remarks are presented in section 7. 


\section{NEW GENERALIZED EXPONENTIAL DISTRIBUTION}

By using the exponentiated-G and EG families, we proposed a three-parameter new generalized (NG) class of distributions as

$$
F(x)=H(x)^{\alpha}\left[1-\bar{H}(x)^{\beta}\right]^{\gamma},
$$

Further, by using the exponential distribution with unity scale parameter $(\vartheta=1)$ in Eq.(2.1), We developed new generalized exponential (NGE) distribution as

$$
F(x)=\left(1-\mathrm{e}^{-x}\right)^{\alpha}\left(1-\mathrm{e}^{-\beta x}\right)^{\gamma}
$$

Hereafter, we assume $\psi(x)=\left(1-\mathrm{e}^{-x}\right)$ and $\psi(x ; \beta)=\left(1-\mathrm{e}^{-\beta x}\right)$ for simplicity. Then, the corresponding probability density function (pdf) of the NGE distribution is

$$
f(x)=\alpha \mathrm{e}^{-x} \psi(x)^{\alpha-1} \psi(x ; \beta)^{\gamma}+\gamma \beta \mathrm{e}^{-\beta x} \psi(x)^{\alpha} \psi(x ; \beta)^{\gamma-1} .
$$

Henceforth, If $X$ is a random variable that follows the pdf (2.3), It can be defined by $X \sim \operatorname{NGE}(\alpha, \beta, \gamma)$. For simplicity, we can consider $f(x)=f(x ; \alpha, \beta, \gamma)$. The NGE distribution shares a interesting physical interpretation. If we consider $\alpha$ and $\beta$ are positive integers and $\gamma=1$, the cdf of the NGE distribution is product of the distributions of maximum and minimum of a sample of size $\alpha$ and $\beta$, respectively, from the exponential distribution. In other words, when the components are identical and independently distributed as exponential distribution $G(x)$, for $\gamma=1$, the NGE model is a product of Weibull competing risk model $\left[1-\prod_{i=1}^{n}\left(1-G_{i}(x)\right)\right]$ and multiplicative Weibull model $\prod_{i=1}^{n} G_{i}(x)$. These models are among the various extensions of the Weibull distribution discussed by [14]. The NGE distribution with three shape parameters is a flexible model which has some popular sub-models as particular cases are given in Table 1.

\begin{tabular}{|c|c|c|c|}
\hline$\alpha$ & $\beta$ & $\gamma$ & Sub-model \\
\hline 1 & 1 & 0 & Exponential distribution \\
$\alpha$ & 1 & 0 & Exponentiated exponential distribution \\
0 & $\beta$ & $\gamma$ & Exponentiated generalized exponential distribution \\
\hline
\end{tabular}

TABLE 1. Sub-model of the NGE distribution 


\section{SOME BASIC PROPERTIES}

3.1. The mode. The silent features of a distribution can be investigated by the first two derivatives of the pdf. Therefore, we differentiate (2.3) with respect to $x$ and equate to zero, namely

$$
\begin{aligned}
f^{\prime}(x) & =\alpha \mathrm{e}^{-x} \psi(x)^{\alpha-2} \psi(x ; \beta)^{\gamma}\left(\alpha \mathrm{e}^{-x}-1\right)+\gamma \beta^{2} \mathrm{e}^{-\beta x} \psi(x)^{\alpha} \psi(x ; \beta)^{\gamma-2}\left(\gamma \mathrm{e}^{-\beta x}-1\right) \\
& +2 \alpha \beta \gamma \mathrm{e}^{-(\beta+1) x} \psi(x)^{\alpha-1} \psi(x ; \beta)^{\gamma-1}=0 .
\end{aligned}
$$

Therefore, the critical values of $f(x)$ can be obtained from the equation

$$
\alpha\left(\alpha \mathrm{e}^{-x}-1\right)+\frac{2 \alpha \beta \gamma \mathrm{e}^{-\beta x} \psi(x)}{\psi(x ; \beta)}=\beta^{2} \gamma \mathrm{e}^{-(\beta-1) x}\left(1-\gamma \mathrm{e}^{-\beta x}\right) .
$$

If the root of Eq.(3.1) is $x=x_{0}$, then the local maximum, local minimum and inflection points obtained when $f^{\prime \prime}\left(x_{0}\right)<0, f^{\prime \prime}\left(x_{0}\right)>0$ and $f^{\prime \prime}\left(x_{0}\right)=0$ respectively. For $\gamma=0$, the mode of $\mathrm{X}$ reduces to $\log \alpha$. For $\alpha=0$, the mode of the density of the NGE model reduces to $\frac{\log \gamma}{\beta}$. For $\beta=1$ the mode becomes $\log (\theta)$, where $\theta=\alpha+\gamma$. For all these three special cases, the NGE distribution becomes the EE distribution. For $\alpha=\gamma=1$, the mode of $\mathrm{X}$ becomes

$$
x=\log \left[\frac{(\beta+1)^{2}}{1+\beta^{2} \mathrm{e}^{-(\beta-1) x}}\right]^{\frac{1}{\beta}}
$$

For $\beta=1$, the last equation reduces to $x=\log (2)$. However, $f^{\prime}(\infty)=0$ and when $x \rightarrow 0$ the limit of $f^{\prime}(x)$ is

$$
\lim _{x \rightarrow 0} f^{\prime}(x)= \begin{cases}0 & \text { if } \alpha+\gamma>2 \\ 2 \beta^{\gamma} & \text { if } \alpha+\gamma=2 \\ \infty & \text { if } \alpha+\gamma<2\end{cases}
$$

\subsection{Density Shapes.}

i. For $\beta=1$ (a) $\alpha+\gamma \leq 1$ (b) $\gamma=0, \alpha \leq 1$, (c) $\alpha=0, \gamma \leq 1$ the NGE density is strictly decreasing.

ii. For $\beta=1$ (a) $\alpha+\gamma>1$ (b) $\gamma=0, \alpha>1$ (c) $\alpha=0, \gamma>1$ the NGE density is uni-modal skewed.

iii. For $\alpha=\beta=\gamma=1$, the NGE density is positively skewed with mode at $x=0.6931$.

iv. For $\alpha=\beta=1, \gamma=0$, the NGE density is reversed type J-shaped (exponential).

v. For $\alpha=0, \beta=\gamma=1$, the NGE density is reversed type J-shaped (exponential).

vi. For $\beta=1$, the shape of the NGE density remains similar by interchanging the values of $\alpha$ and $\gamma$.

vii. For $\alpha+\gamma>1$ and $\beta>0$, the NGE density is unimodel and as the value of $\beta$ increases the density becomes more skewed with longer tails. 
viii. For $\alpha+\gamma \leq 1$ and $\beta>0$, the NGE density is reversed J-shaped with longer tails and when $\beta \rightarrow \infty$ the tails become shorter.

ix. For $\alpha=1, \gamma=1$ and $\beta>0$, the NGE density is unimodal and skewed and when $\beta \rightarrow \infty$ the skewness increases.

$\mathrm{x}$. For $\alpha>1, \gamma>1$ and $\beta>0$, the NGE density is unimodal and positively skewed, and for $\beta>2$, the mode of the NGE density does not significantly change.

xi. For $\beta<1, \gamma<1$ and $0<\alpha \leq 1(\alpha>1)$, the NGE density is reversed J-shaped with shorter height (unimodal positively skewed with increasing mode).

xii. For $\beta>1, \gamma>1$ and $0<\alpha \leq 1(\alpha>1)$, the NGE density is unimodal positive skewed with sharp peak (unimodal positive skewed with shorter height), and when $\alpha \rightarrow \infty$, the mode of the density increases with heavy tails.

xiii. For $\alpha<1, \beta<1$ and $0<\gamma \leq 1(\gamma>1)$, the NGE density is reversed J-shaped (unimodal positive skewed with increasing mode).

xiv. For $\alpha>1, \beta>1$ and $0<\gamma<\infty$, an insignificant change is noted in the NGE density.

We displayed explanatory graphs of the densities of $\mathrm{X}$ for different specific parameter values in Figure 1. The density of the $X$ takes various forms for different specific parameter values of the NGE distribution. It can be noted that the NGE density is more flexible than its competitors. So, the proposed distribution can effectively be used for modeling the positive data.

3.3. Hazard rate function (hrf). An interesting feature of the proposed model that it can have the monotonically increasing, decreasing, bathtub and uni-modal (reversed bathtub) hrf for different parameter values. The hrf of the NGE distribution is

$$
h(x)=\frac{\alpha \mathrm{e}^{-x} \psi(x)^{\alpha-1} \psi(x ; \beta)^{\gamma}+\gamma \beta \mathrm{e}^{-\beta x} \psi(x)^{\alpha} \psi(x ; \beta)^{\gamma-1}}{1-\left[\psi(x)^{\alpha} \psi(x ; \beta)^{\gamma}\right]} .
$$

For $\gamma=0$ or $\beta=1$, the hrf of $\mathrm{X}$ reduces to the hrf of the EE distribution and For $\alpha=1$ and $\gamma=0$, the hrf becomes constant.

In Fig.2, we provide the visual representation of the hrfs of the $\mathrm{X}$ which illustrate important characteristics of the proposed model. It can be seen that the hrf is monotonically increasing, decreasing, uni-model, decreasing-increasing-decreasing, increasing-decreasing-increasing and constant. So, the proposed distribution is quite flexible to model the data which have different hrfs.

\subsection{Shapes of the hrf.}

i. For $\beta=1$ (a) $\alpha+\gamma \leq 1$ (b) $\gamma=0, \alpha \leq 1$ (c) $\alpha=0, \gamma \leq 1$, the hrf of $\mathrm{X}$ is decreasing from $\infty$ to $\vartheta$.

ii. For $\beta=1$ (a) $\alpha+\gamma>1$ (b) $\gamma=0, \alpha>1$, (c) $\alpha=0, \gamma>1$, the hrf of $\mathrm{X}$ is increasing from 0 to $\vartheta$.

iii. For $\beta=1$ (a) $\alpha+\gamma=1$ (b) $\gamma=0, \alpha=1$ (c) $\alpha=\gamma=1$, the hrf of $\mathrm{X}$ is constant $\vartheta=1$. 
(a)

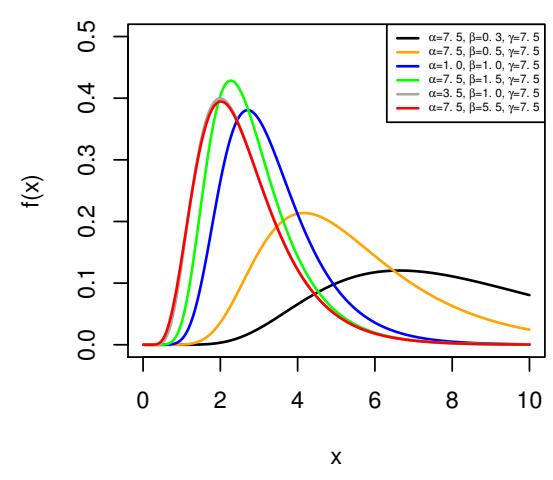

(c)

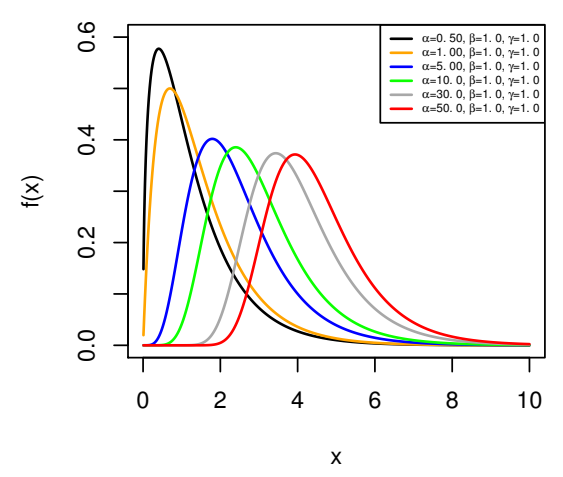

(b)

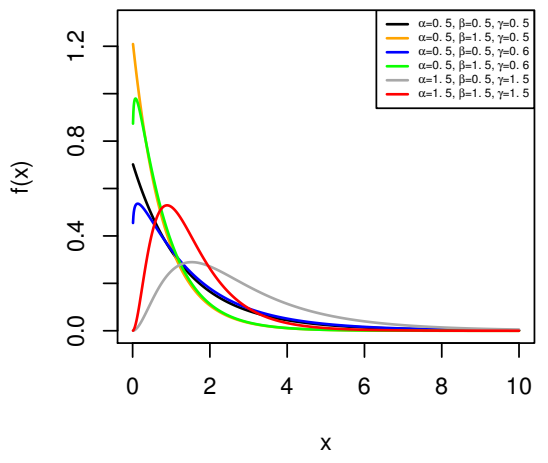

(d)

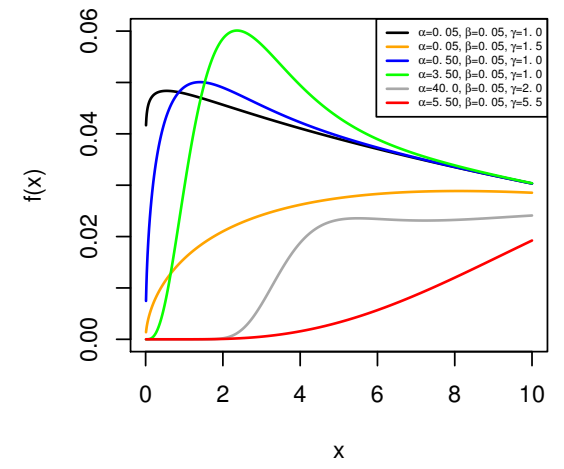

Figure 1. Plots of the density (2.3) for some specific parameter values.

iv. For $\alpha+\gamma>1$ and $0<\beta<1$ and $1<\beta<\infty$, the shape of the hrf of $\mathrm{X}$ is reversed bathtub.

v. For $\alpha+\gamma \leq 1$ and $0<\beta<1$ and $1<\beta<\infty$, the hrf of $\mathrm{X}$ is bathtub type.

vi. For $\alpha \rightarrow 0, \beta>1.5, \gamma<1$, the hrf of $\mathrm{X}$ is decreasing-increasing-decreasing towards $\vartheta=1$.

vii. For $\alpha \rightarrow 0, \beta>1.5, \gamma>1$, the hrf of $\mathrm{X}$ is uni-model.

3.5. Asymptotics of the density and hrf. The asymptotics of (2.3) and (3.2) are $(\alpha+\gamma) \beta^{\gamma} x^{\alpha+\gamma-1}$ as $x \rightarrow 0$, further $f(x) \sim \alpha e^{-x}+\beta \gamma \mathrm{e}^{-\beta x}$ and $h(x) \sim \frac{\alpha \mathrm{e}^{-x}+\beta \gamma \mathrm{e}^{-\beta x}}{\alpha(\alpha-2) \mathrm{e}^{-x}+2 \alpha \beta \gamma \mathrm{e}^{-(\beta+1) x}+\gamma \beta^{2} \mathrm{e}^{-\beta x}}$ as $x \rightarrow \infty$. It is noted that the tail behaviors of both equations are polynomial and also exponential.

Theorem 3.1. Let $X \sim N G E(\alpha, \beta, \gamma)$. Then the asymptotic behaviors of (2.3) and (3.2) are:

$$
\lim _{x \rightarrow 0} f(x)= \begin{cases}0 & \text { if }(\alpha+\gamma)>1 \\ \beta^{\gamma} & \text { if }(\alpha+\gamma)=1 \\ \infty & \text { if }(\alpha+\gamma)<1\end{cases}
$$



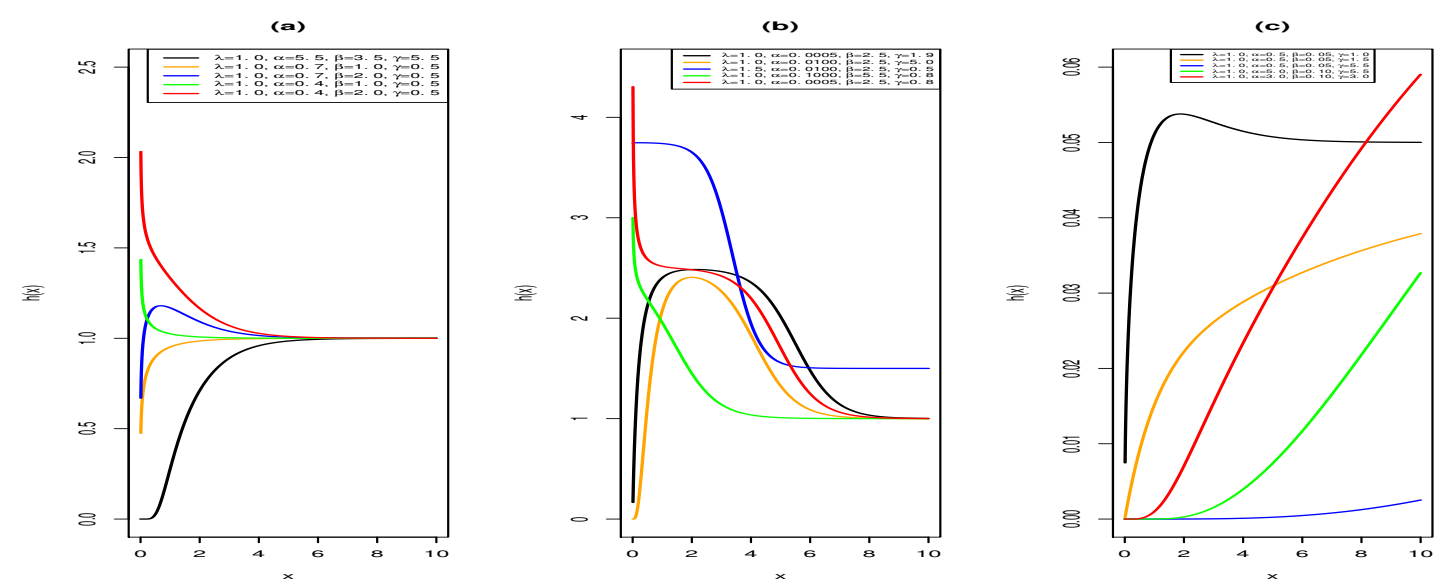

Figure 2. Plots of the hrf of $\mathrm{X}$ for different selected values of the shape parameters when $\vartheta=1$.

and

$$
\lim _{x \rightarrow \infty} f(x)=\lim _{x \rightarrow \infty} h(x)=0 .
$$

Proof. $\left(1-\mathrm{e}^{-x}\right)=x$ when $x \rightarrow 0$, and $\mathrm{e}^{-x} \rightarrow 0$ as $x \rightarrow \infty$. The limits of $f(x)$ can then be obtained. Similarly, when $x \rightarrow 0$, the limiting behavior of $h(x)$ is the same as the limit of $f(x)$. As $x \rightarrow \infty$ both numerator and denominator become 0 of the hrf of $\mathrm{X}$. Thus, the hrf is indeterminate. We evaluate the limit by using L'Hôpital's rule. So, it completes the proof.

3.6. Reversed hazard rate function ( $r h r f)$. The rhrf of the $\mathrm{X}$ is

$$
r(x)=\frac{\alpha \mathrm{e}^{-x}}{\psi(x)}+\frac{\gamma \beta \mathrm{e}^{-\beta x}}{\psi(x ; \beta)} .
$$

Equation (3.3) shows that the rhrf of $\mathrm{X}$ is a sum of the proportional rhrf of the EE and EGE distributions. For $\gamma=0$, Eq.(3.3) becomes

$$
r(x)=\frac{\alpha \mathrm{e}^{-x}}{\psi(x)} .
$$

Therefore, rhrf of $\mathrm{X}$ is proportionally equals to the rhrf of the exponential distribution.

\section{LiNEAR REPRESENTATION}

We use the power series for any non-integer real $\theta$ as

$$
(1-z)^{\theta}=\sum_{k=0}^{\infty} \frac{(-1)^{k} \Gamma(\theta)}{\Gamma(\theta-k) k !} z^{i}, \forall|z|<1
$$


By using (4.1) in (2.2), we obtain

$$
F(x)=\sum_{j=0}^{\infty} \tau_{j} \psi(x)^{\alpha+j}
$$

where the coefficients $\tau_{j}=\tau_{j}(\beta, \gamma)$ are

$$
\tau_{j}=\frac{(-1)^{j} \Gamma(\gamma+1)}{j !} \sum_{k=0}^{\infty} \frac{(-1)^{k} \Gamma(k \beta+1)}{\Gamma(k \beta-j+1) \Gamma(\gamma-k+1) k !}
$$

We can rewrite $(4.2)$ as

$$
F(x)=\sum_{j=0}^{\infty} \tau_{j} W_{\alpha+j}(x),
$$

where $W_{\alpha+j}(x)=\psi(x)^{\alpha+j}$ is the cdf of the EE distribution with power parameter is $\alpha+j$. By differentiating (4.3), a simple linear representation of (2.3) is

$$
f(x)=\sum_{j=0}^{\infty} \tau_{j} w_{\alpha+j}(x),
$$

where $w_{\alpha+j}(x)=(\alpha+j) \psi(x)^{\alpha+j-1} \mathrm{e}^{-x}$ is the pdf of the EE distribution with power parameter is $\alpha+j$. Equation(4.4) informs that the pdf of the NGE distribution is linear mixture of well-known EE densities. Hence, various important statistical properties of the NGE distribution can be developed from simple properties of the EE distribution.

\section{Mathematical Properties}

5.1. Generation function. We can use the moment generating function (mgf) to characterize the distributions. It is also used for generating the moments of a distribution.

Theorem 5.1. The mgf of $X$ is defined by

$$
M(t)=\sum_{j=0}^{\infty} \tau_{j} \frac{\Gamma(\alpha+j+1) \Gamma(1-t)}{\Gamma(\alpha-t+j+1)}
$$

Proof. By using (4.4), we have

$$
M(t)=\sum_{j=0}^{\infty} \tau_{j}(\alpha+j) \int_{0}^{\infty} \mathrm{e}^{(t-1) x} \psi(x)^{\alpha+j-1} d(x) .
$$

Setting $\mathrm{e}^{-x}=u, M(t)$ can be written as

$$
M(t)=\sum_{j=0}^{\infty} \tau_{j}(\alpha+j) \int_{0}^{1} u^{-t}(1-u)^{\alpha+j-1} d x,
$$

which after simplification leads to (5.1). 


$$
M(t)=\sum_{j=0}^{\infty} \tau_{j} \frac{\Gamma(\alpha+j+1) \Gamma(1-t)}{\Gamma(\alpha-t+j+1)}
$$

If $\gamma=0$ and $\vartheta=1$, The mgf of the NGE distribution reduces to the mgf of the EE distribution given by $[7]$.

5.2. Moments. Moments play important role to know about the different aspects of a probability distribution such as the central tendency and variation of a distribution. Furthermore, the moments can also be employed to examine the skewness and kurtosis of a probability distribution.

We can write from (4.4), setting $\mathrm{e}^{-x}=u$, and after some algebra

$$
\mu_{r}=\left.(-1)^{r} \sum_{j=0}^{\infty}(\alpha+j) \tau_{j} \frac{\partial^{r}}{\partial p^{r}} B(\alpha+j, p+1)\right|_{p=0}
$$

Alternatively, we obtain another expression

$$
\mu_{r}=r ! \sum_{j=0}^{\infty}(\alpha+j) \tau_{j} \sum_{p=0}^{\infty} \frac{(-1)^{p}\left(\begin{array}{c}
\alpha+j-1 \\
p
\end{array}\right)}{(p+1)^{r+1}} .
$$

A comparison is also made of the mean-variance for different parameter values in Figure 3. The numerical values of the first two moments, standard deviation, median, mode, skewness and kurtosis for various specific parameter values of $\mathrm{X}$ are given in Table 2. For some different specific parameter values of the $\mathrm{X}$, the skewness and kurtosis measures are illustrated in Figure 4. It examined that these measures depend only on the shape parameters. 

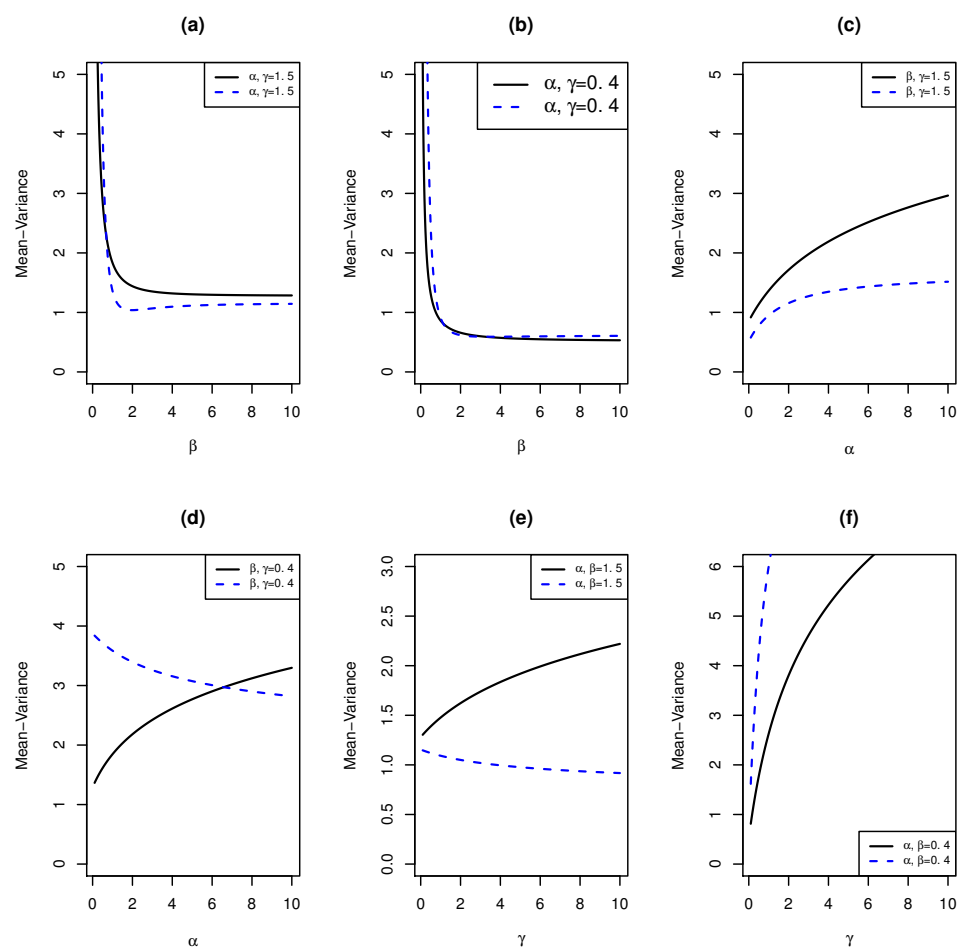

FiguRE 3. Plots for the mean and variation of $\mathrm{X}$, where black line represent the mean of $\mathrm{X}$ and doted lines represent the variance of $\mathrm{X}$ when $\vartheta=1$. 
(a) $\beta=4.5$

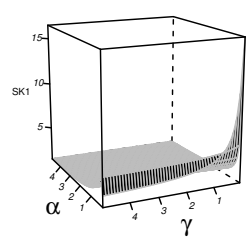

(d) $\beta=4.5$

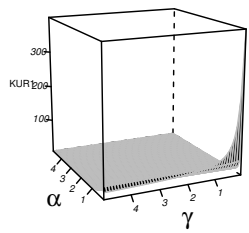

(b) $\beta=0.5$

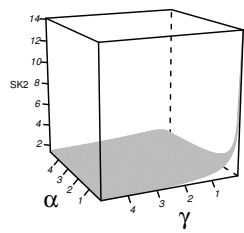

(e) $\beta=0.5$

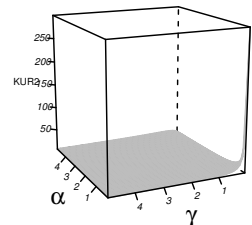

(c) $\beta=0.01$

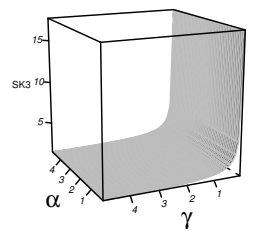

(f) $\beta=0.01$

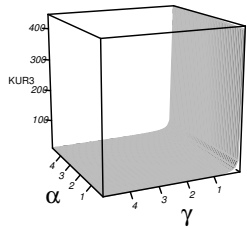

Figure 4. Plots of skewness $(\mathrm{a}, \mathrm{b}, \mathrm{c})$ and kurtosis $(\mathrm{d}, \mathrm{e}, \mathrm{f})$ of the $\mathrm{X}$ when $\vartheta=1$.

5.3. Quantile function. If $X \sim \operatorname{NGE}(\alpha, \beta, \gamma)$, the quantile function of $\mathrm{X}$ is determined numerically from

$$
\left[\psi(x)^{\alpha} \psi(x ; \beta)^{\gamma}\right]=u
$$

First, second and third quartile of the distribution can be calculated by setting $u=0.25,0.50$ and 0.75 , respectively.

5.4. Mean deviations. The mean deviations can be used to compute the variation of $\mathrm{X}$. Then, the mean deviations about the average value (mean) and median can be determined by

$$
\phi_{1}(x)=2 \mu_{1} F(\mu)-2 m\left(\mu_{1}\right) \text { and } \phi_{2}(x)=\mu_{1}-2 m(M) \text {, }
$$

respectively, here $\mu_{1}$ is the mean of $\mathrm{X}$ calculated from (5.2) and $M$ is its median. The first incomplete moment is required for measuring (5.3). It is defined by $m(z)=\int_{-\infty}^{z} w f(w) d w$. Now by using (4.4) and (4.1) $m(z)$ reduces to

$$
m(z)=\sum_{j=0}^{\infty}(\alpha+j) \tau_{j} \sum_{p=0}^{\infty}(-1)^{p}\left(\begin{array}{c}
\alpha+j-1 \\
p
\end{array}\right) \int_{0}^{z} x \mathrm{e}^{-(p+1) x} d x
$$


TABLE 2. The values of first two moments, standard deviation, median, mode, skewness and kurtosis of $X$.

\begin{tabular}{llllllll}
\hline$(\alpha, \beta, \gamma)$ & $E(X)$ & $E\left(X^{2}\right)$ & $S D$ & Median & Mode & Sk & Kur \\
\hline$(1,0.3,1)$ & 3.6 & 23 & 3.2 & 2.59 & 1.14 & 2.1 & 9.7 \\
$(1,0.5,1)$ & 2.3 & 9.1 & 1.9 & 1.82 & 0.95 & 2.0 & 9.3 \\
$(1,1,1)$ & 1.5 & 3.5 & 1.1 & 1.23 & 0.69 & 1.6 & 7.1 \\
$(1,1.5,1)$ & 1.3 & 2.6 & 0.98 & 1.02 & 0.6 & 1.8 & 8.2 \\
$(1,2,1)$ & 1.2 & 2.3 & 0.96 & 0.91 & 0.47 & 2.0 & 9.3 \\
$(1,5,1)$ & 1.0 & 2.0 & 0.98 & 0.72 & 0.26 & 2.1 & 9.5 \\
$(0.5,0.5,0.5)$ & 1.5 & 5.1 & 1.7 & 1.00 & $1.6 \times 10^{-10}$ & 2.4 & 12 \\
$(1,0.5,0.5)$ & 1.8 & 5.8 & 1.6 & 1.29 & 0.49 & 2.3 & 12 \\
$(2,0.5,0.5)$ & 2.1 & 7.0 & 1.6 & 1.68 & 1.03 & 2.2 & 11 \\
$(5,0.5,0.5)$ & 2.7 & 9.6 & 1.5 & 2.34 & 1.82 & 2.0 & 10 \\
$(2,2,2)$ & 1.6 & 3.8 & 1.1 & 1.40 & 0.98 & 1.7 & 7.7 \\
$(3,2,2)$ & 1.9 & 5.0 & 1.1 & 1.68 & 1.24 & 1.5 & 6.9 \\
$(5,2,2)$ & 2.3 & 6.8 & 1.2 & 2.09 & 1.66 & 1.4 & 6.3 \\
$(1.5,1.5,0.5)$ & 1.4 & 3.0 & 1.1 & 1.23 & 0.62 & 1.7 & 7.6 \\
$(1.5,1.5,1.5)$ & 1.6 & 3.5 & 1.0 & 1.32 & 0.89 & 1.6 & 7.5 \\
$(1.5,1.5,5.5)$ & 2.0 & 4.8 & 0.98 & 1.76 & 1.42 & 1.5 & 7.2 \\
$(0.5,1.5,0.4)$ & 0.8 & 1.4 & 0.86 & 0.52 & $2.3 \times 10^{-9}$ & 2.3 & 11 \\
$(0.5,1.5,1.5)$ & 1.1 & 2.1 & 0.88 & 0.92 & 0.51 & 1.8 & 8.7 \\
$(0.5,1.5,5.5)$ & 1.7 & 3.7 & 0.89 & 1.54 & 1.24 & 1.5 & 7.0 \\
$(1.5,0.5,0.5)$ & 1.9 & 4.3 & 1.4 & 1.21 & 0.57 & 2.3 & 13 \\
$(1.5,0.5,1.5)$ & 2.9 & 12 & 2.0 & 2.37 & 1.53 & 1.8 & 8.2 \\
$(1.5,0.5,5.5)$ & 4.8 & 29 & 2.4 & 4.32 & 3.46 & 1.4 & 6.1 \\
$(0.5,0.5,0.5)$ & 1.5 & 5.1 & 1.7 & 1.00 & $1.6 \times 10^{-10}$ & 2.4 & 12 \\
$(0.5,0.5,1)$ & 2.2 & 8.6 & 2.0 & 1.63 & 0.62 & 2.0 & 9.1 \\
$(0.5,0.5,1.5)$ & 2.7 & 12 & 2.1 & 2.14 & 1.14 & 1.8 & 7.9 \\
\hline & & & & & & &
\end{tabular}

By setting $(p+1) x=u$ and using $\Gamma(a, x)=\int_{0}^{\infty} z^{a-1} e^{-z} d z$, we obtain

$$
m(z)=\sum_{j=0}^{\infty}(\alpha+j) \tau_{j} \sum_{p=0}^{\infty} \frac{(-1)^{p}\left(\begin{array}{c}
\alpha+j-1 \\
p
\end{array}\right) \Gamma(2,(p+1) z)}{(p+1)^{2}} .
$$


The values of mean deviations of the NGE distribution about the mean and median can be obtained by using Eq.(5.4). We can also use (5.4) to construct the Bonferroni and Lorenz curves which have much importance in economics, renewal theory and reliability theory. For a given probability $p$, we can define these curves by $B(p)=m(q) /\left(p \mu_{1}\right)$ and $L(p)=m(q) / \mu_{1}$, where $q=Q(p)$ is the quantile function of $\mathrm{X}$ at $p$ and $\mu_{1}=E(X)$.

\section{Estimation AND INFERENCE}

There are different methods of point estimation but the maximum likelihood is the famous technique to calculate the point estimates of the unknown quantities of a distribution. The maximum likelihood estimates (MLEs) have some nice and useful characteristics. Moreover, we can use the MLEs to calculate the confidence intervals for the unknown parameters of a distribution. Thus, we choose the technique of maximum likelihood to estimate the unknown quantities of the $\mathrm{X}$ from the given sample. Let $x_{1}, x_{2}, \ldots, x_{n}$ be observed sample from (2.3) and $\Omega=(\alpha, \beta, \gamma)^{T}$ be a vector of parameters of X. Then, we will define the log-likelihood function by

$$
\begin{aligned}
l(\Omega) & =(\alpha-1) \sum_{i=1}^{n} \log \left(\psi\left(x_{i}\right)\right)+\gamma \sum_{i=1}^{n} \log \left(\psi\left(x_{i} ; \beta\right)\right)-\sum_{i=1}^{n} x_{i} \\
& +\sum_{i=1}^{n} \log \left\{\alpha \psi\left(x_{i} ; \beta\right)+\beta \gamma \mathrm{e}^{-(\beta-1) x_{i}} \psi\left(x_{i}\right)\right\}-\sum_{i=1}^{n} \log \left(\psi\left(x_{i} ; \beta\right)\right) .
\end{aligned}
$$

The elements of the score are

$$
\begin{aligned}
& \frac{\partial l(\Omega)}{\partial \alpha}=\sum_{i=1}^{n} \log \left(\psi\left(x_{i}\right)\right)+\sum_{i=1}^{n} \frac{\psi\left(x_{i} ; \beta\right)}{\nu\left(x_{i}\right)} \\
& \frac{\partial l(\Omega)}{\partial \beta}=(\gamma-1) \sum_{i=1}^{n} \frac{x_{i} \mathrm{e}^{-\beta x_{i}}}{\psi\left(x_{i} ; \beta\right)}+\sum_{i=1}^{n} \frac{\alpha x_{i} \mathrm{e}^{-\beta x_{i}}+\gamma \mathrm{e}^{-(\beta-1) x_{i}} \psi\left(x_{i}\right) \psi\left(x_{i} ; \beta\right)}{\nu\left(x_{i}\right)} \\
& \frac{\partial l(\Omega)}{\partial \gamma}=\sum_{i=1}^{n} \log \left(\psi\left(x_{i} ; \beta\right)\right)+\beta \sum_{i=1}^{n} \frac{\mathrm{e}^{-(\beta-1) x_{i}} \psi\left(x_{i}\right)}{\nu\left(x_{i}\right)}
\end{aligned}
$$

where $\nu\left(x_{i}\right)=\alpha \psi\left(x_{i} ; \beta\right)+\beta \gamma \mathrm{e}^{-(\beta-1) x_{i}} \psi\left(x_{i}\right)$. The MLEs $\hat{\Omega}$ of $\Omega$ can be calculated by solving the above equations simultaneously after equate these equations to zero. There is no analytical solution for these equations. Therefore, we will solve the score equations numerically by using some nonlinear optimization algorithm in open source software environment for statistical programming $\mathrm{R}$.

To determine the test of hypothesis and find the interval estimate of the unknown parameters of X, we require the $3 \times 3$ observed information matrix 


$$
J(\Omega)=\left[\begin{array}{ccc}
J_{\alpha, \alpha} & J_{\alpha, \beta} & J_{\alpha, \gamma} \\
J_{\alpha, \beta} & J_{\beta, \beta} & J_{\beta, \gamma} \\
J_{\alpha, \gamma} & J_{\beta, \gamma} & J_{\gamma, \gamma}
\end{array}\right]
$$

Under the relevant regularity conditions, the asymptotic distribution of $\sqrt{n}(\hat{\Omega}-\Omega)$ follows multivariate $N_{3}\left(0, J(\hat{\Omega})^{-1}\right)$ which can be employed to establish the confidence intervals for the unknown parameters of the X. Here, we consider $J(\hat{\Omega})$ as the observed information matrix which is evaluated at $\Omega=\hat{\Omega}$.

6.1. Application. In the following important section, we apply the proposed model to real data sets to highlight the capability of the NGE distribution. Here, we compare the EGE, EE and BE distributions with the NGE distribution. The study is conducted in statistical computing R developed and maintained by [20]. First, we provide the description of two real data sets. Then, for each model, We calculate the MLEs of the unknown paramaters and their crossponding standard errors by using the $\mathrm{R}$ package AdequacyModel [4]. The proposed distribution is compared with its competing models by using the well-known statistics in literature like the Cramér-von Mises $\left(\mathrm{W}^{*}\right)$, Anderson-Darling $\left(\mathrm{A}^{*}\right)$, Bayesian Information Criterion (BIC), Akaike Information Criterion (AIC) and Kolmogrov-Smirnov (K-S). Generally, the fit is better if the values of these statistics are small.

The first real data is repair times of 46 failures in (hours) of an airborne communications receiver is analysed by [2] is: $0.2,0.3,0.5,0.5,0.5,0.5,0.6,0.6,0.7,0.7,0.7,0.8,0.8,1.0,1.0,1.0,1.0,1.1,1.3,1.5,1.5$, $1.5,1.5,2.0,2.0,2.2,2.5,2.7,3.0,3.0,3.3,3.3,4.0,4.0,4.5,4.7,5.0,5.4,5.4,7.0,7.5,8.8,9.0,10.3,22.0$, 24.5 .

The second data set have 128 observations which represent the remission times (month) of patients of bladder cancer and studied by [15] is: $0.08,0.20,0.40,0.50,0.51,0.81,0.90,1.05,1.19,1.26,1.35,1.40,1.46$, $1.76,2.02,2.02,2.07,2.09,2.23,2.26,2.46,2.54,2.62,2.64,2.69,2.69,2.75,2.83,2.87,3.02,3.25,3.31,3.36$, $3.36,3.48,3.52,3.57,3.64,3.70,3.82,3.88,4.18,4.23,4.26,4.33,4.34,4.40,4.50,4.51,4.87,4.98,5.06,5.09$, $5.17,5.32,5.32,5.34,5.41,5.41,5.49,5.62,5.71,5.85,6.25,6.54,6.76,6.93,6.94,6.97,7.09,7.26,7.28,7.32$, $7.39,7.59,7.62,7.63,7.66,7.87,7.93,8.26,8.37,8.53,8.65,8.66,9.02,9.22,9.47,9.74,10.06,10.34,10.66$, $10.75,11.25,11.64,11.79,11.98,12.02,12.03,12.07,12.63,13.11,13.29,13.80,14.24,14.76,14.77,14.83$, $15.96,16.62,17.12,17.14,17.36,18.10,19.13,20.28,21.73,22.69,23.63,25.74,25.82,26.31,32.15,34.26$, $36.66,43.01,46.12,79.05$. 
TABle 3. Descriptive statistics for the data sets.

\begin{tabular}{ccc}
\hline Statistic & First data set & Second data set \\
\hline $\mathrm{n}$ & 46 & 128 \\
Median & 1.75 & 6.40 \\
Mode & 2.50 & 5.0 \\
Mean & 3.61 & 9.37 \\
Variance & 24.45 & 110.43 \\
Skewness & 2.88 & 3.29 \\
Kurtosis & 8.80 & 15.48 \\
\hline
\end{tabular}

TABLE 4. MLEs for the parameters of the NGE, EGE, BE and EE distributions with their corresponding standard errors given in (parentheses) and statistics $\mathrm{A}^{*}$ and $\mathrm{W}^{*}$ for repair times data.

\begin{tabular}{ccccccc}
\hline Model & $\vartheta$ & $\alpha$ & $\beta$ & $\gamma$ & $\mathrm{A}^{*}$ & $\mathrm{~W}^{*}$ \\
\hline NGE & 0.1830 & 0.5212 & 18.0765 & 4.7498 & 02211 & 0.0241 \\
& $(0.05325)$ & $(0.1643)$ & $(7.8974)$ & $(3.1616)$ & & \\
EGE & 0.5189 & 0.5190 & 0.9583 & - & 1.0004 & 0.1442 \\
& $(9.8776)$ & $(9.8776)$ & $(0.1897)$ & & & \\
BE & 0.0688 & 0.9344 & 3.7971 & - & 0.9953 & 0.1435 \\
& $(0.3029)$ & $(0.1726)$ & $(16.5490)$ & & & \\
EE & 0.2694 & 0.9583 & - & - & 1.0004 & 0.1442 \\
& $(0.0544)$ & $(0.1897)$ & & & & \\
\hline
\end{tabular}

TABLE 5. Other statistics to compare the models for the repair times data.

\begin{tabular}{cccccc}
\hline Model & $-\mathcal{L L}$ & AIC & BIC & K-S & $\begin{array}{c}\text { p-value } \\
(\mathrm{K}-\mathrm{S})\end{array}$ \\
\hline NGE & 98.8585 & 205.7169 & 213.0315 & 0.0635 & 0.9925 \\
EGE & 104.9829 & 215.9658 & 221.4517 & 0.1520 & 0.2385 \\
BE & 104.9368 & 215.8735 & 221.3594 & 0.1460 & 0.2804 \\
EE & 104.9829 & 213.9658 & 217.6231 & 0.1520 & 0.2385 \\
\hline
\end{tabular}


TABLE 6. MLEs for the parameters of the NGE, EGE, BE and EE distributions with their corresponding standard errors given in (parentheses) and statistics $\mathrm{A}^{*}$ and $\mathrm{W}^{*}$ for remission times data.

\begin{tabular}{ccccccc}
\hline Model & $\vartheta$ & $\alpha$ & $\beta$ & $\gamma$ & $\mathrm{A}^{*}$ & $\mathrm{~W}^{*}$ \\
\hline NGE & 0.1969 & 1.3169 & 0.2851 & 0.2003 & 0.1437 & 0.0210 \\
& $(0.0582)$ & $(0.2761)$ & $(0.1148)$ & $(0.2310)$ & & \\
EGE & 0.1370 & 0.8846 & 1.2179 & - & 0.6741 & 0.1122 \\
& $(0.7036)$ & $(4.5442)$ & $(0.1488)$ & & & \\
BE & 0.6435 & 1.4480 & 0.1798 & - & 3.3864 & 0.5264 \\
& $(0.6158)$ & $(0.3292)$ & $(0.1791)$ & & & \\
EE & 0.1213 & 1.2219 & - & - & 0.6733 & 0.1120 \\
& $(0.0139)$ & $(0.1494)$ & & & & \\
\hline
\end{tabular}

TABLE 7. Other statistics to compare the models for the remission times data.

\begin{tabular}{cccccc}
\hline Model & $-\mathcal{L L}$ & AIC & BIC & K-S & $\begin{array}{c}\text { p-value } \\
(\mathrm{K}-\mathrm{S})\end{array}$ \\
\hline $\mathrm{NGE}$ & 409.7355 & 827.4711 & 838.8792 & 0.0381 & 0.9925 \\
$\mathrm{EGE}$ & 413.0776 & 832.1552 & 840.7113 & 0.0725 & 0.5113 \\
$\mathrm{BE}$ & 412.3440 & 830.6880 & 839.2441 & 0.0664 & 0.6244 \\
$\mathrm{EE}$ & 413.0779 & 830.1560 & 835.8600 & 0.0728 & 0.5053 \\
\hline
\end{tabular}


(a)

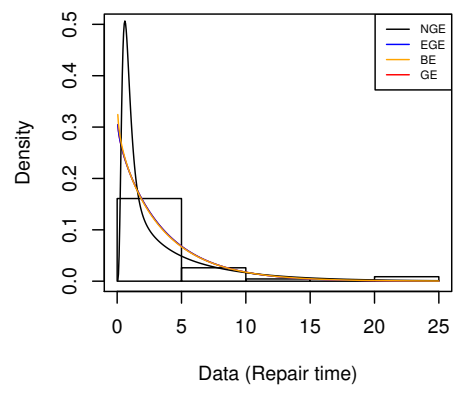

(c)

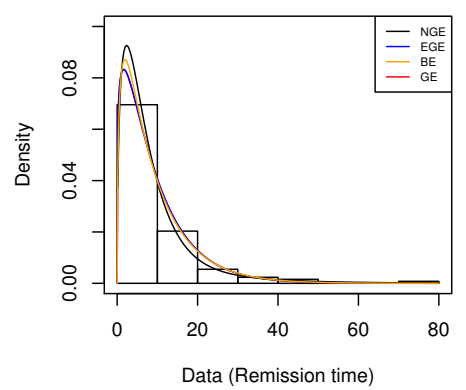

(b)

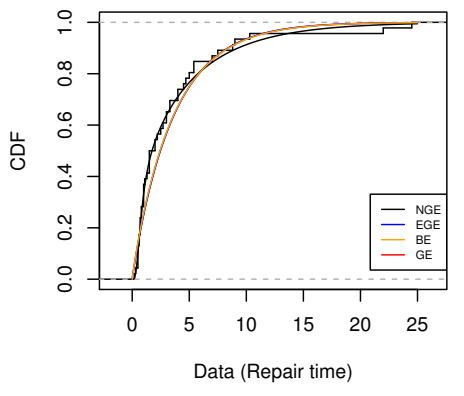

(d)

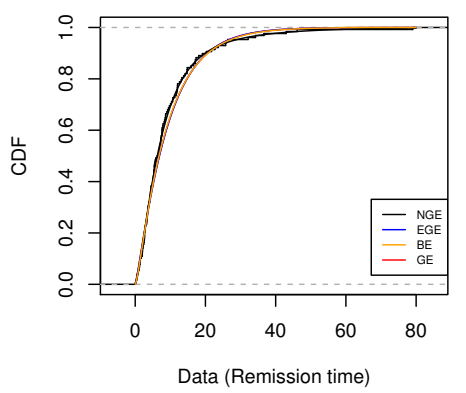

Figure 5. For repair times data: (a) Plots of estimated density functions (b) estimated cdfs of the NGE and their competing models EGE, BE and EE, For remission times data: (c) Plots of estimated densities (d) estimated cdfs of the NGE, EGE, BE and EE distributions. 

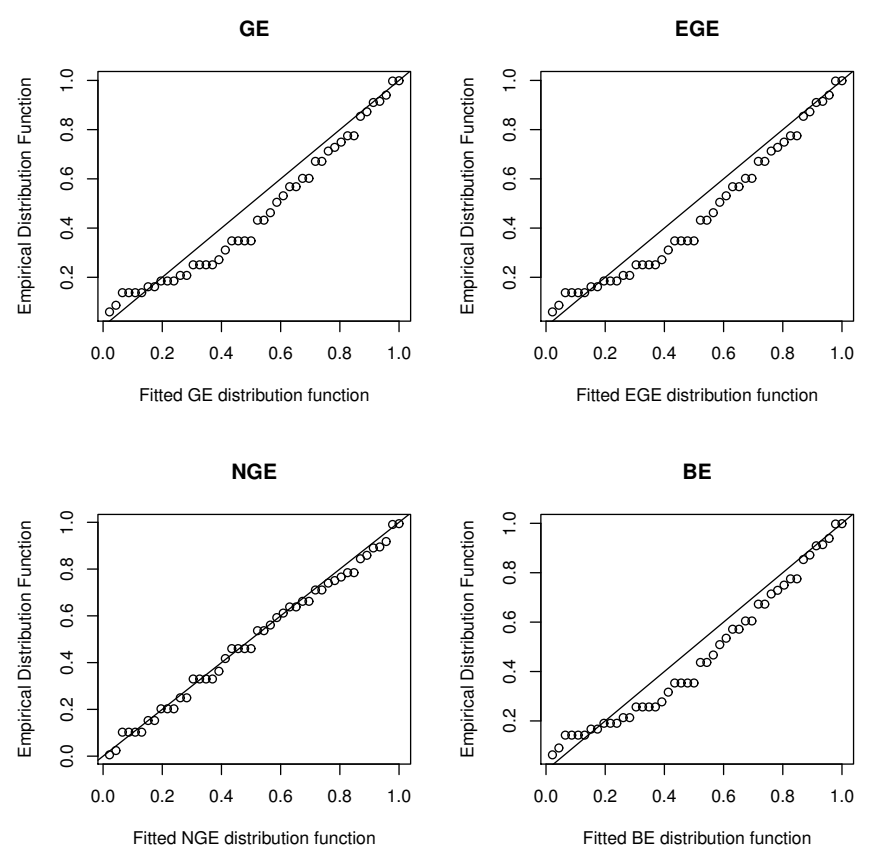

Figure 6. Fitted and empirical distribution functions for repair times data.
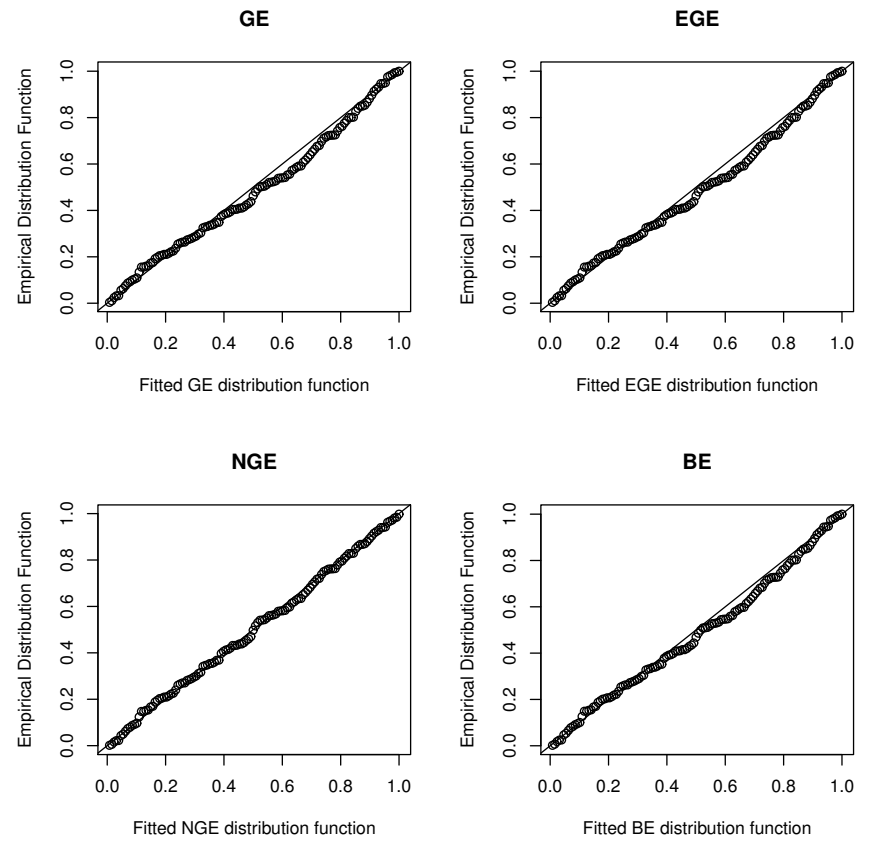

Figure 7. Fitted and empirical distribution functions for remission times data. 


\section{FinAL REMARKS}

We develop a new continuous probability distribution, named new generalized exponential (NGE) distribution. We study its properties theoretically and numerically. We provide the linear presentation of the density function which is quite useful to drive the simple expressions of several statistical properties of the proposed distribution. Moreover, the simple expressions are derived for some properties of the X. The parameters of the $\mathrm{X}$ are estimated by the technique of maximum likelihood. To compare the proposed model with other models, we apply these models to two sets of real data from different fields of science such as biology and engineering and fit is examined by using well-known statistics. We conclude that the NGE distribution fit better than the EGE, BE and EE distributions. We are hopeful that the NGE distribution is another very useful distribution to study the problems in several fields such as economics, biology, engineering and reliabiliy theory.

Conflicts of Interest: The author(s) declare that there are no conflicts of interest regarding the publication of this paper.

\section{REFERENCES}

[1] W. Barreto-Souza, A.H.S. Santos, G.M. Cordeiro, The beta generalized exponential distribution, J. Stat. Comput. Simul. 80 (2010), 159-172.

[2] R.S. Chhikara, J.L. Folks, The Inverse Gaussian Distribution as a Lifetime Model, Technometrics. 19 (1977), $461-468$.

[3] G.M. Cordeiro, E.M. Ortega, D.C. da Cunha, The exponentiated generalized class of distributions, J. Data Sci. 11 (2013), 1-27.

[4] P. R. Diniz Marinho, M. Bourguignon, and C. R. Barros Dias, AdequacyModel: Adequacy of Probabilistic Models and General Purpose Optimization, R package version 2.0.0. 2016. https://cran.r-project.org/package=AdequacyModel

[5] R.C. Gupta, P.L. Gupta, R.D. Gupta, Modeling failure time data by lehman alternatives, Commun. Stat. Theory Methods. 27 (1998), 887-904.

[6] R.D. Gupta, D. Kundu, Theory \& Methods: Generalized exponential distributions, Aust. N.Z. J. Stat. 41 (1999), $173-188$.

[7] R.D. Gupta, D. Kundu, Exponentiated exponential family: an alternative to gamma and weibull distributions, Biom. J. 43 (2001), 117-130.

[8] R.D. Gupta, Debasis. Kundu, Generalized exponential distribution: different method of estimations, J. Stat. Comput. Simul. 69 (2001), 315-337.

[9] R.D. Gupta, D. Kundu, Closeness of Gamma and Generalized Exponential Distribution, Commun. Stat. Theory Methods. 32 (2003), 705-721.

[10] R.D. Gupta, D. Kundu, Discriminating between Weibull and generalized exponential distributions, Comput. Stat. Data Anal. 43 (2003), 179-196.

[11] R.D. Gupta, D. Kundu, On the comparison of Fisher information of the Weibull and GE distributions, J. Stat. Plan. Inference. 136 (2006), 3130-3144.

[12] R.D. Gupta, D. Kundu, Generalized exponential distribution: Existing results and some recent developments, J. Stat. Plan. Inference. 137 (2007), 3537-3547. 
[13] D. Kundu, R.D. Gupta, A. Manglick, Discriminating between the log-normal and generalized exponential distributions, J. Stat. Plan. Inference. 127 (2005), 213-227.

[14] C.D. Lai, D.N.P. Murthy, M. Xie, Weibull distributions, WIREs Comput. Stat. 3 (2011), $282-287$.

[15] E.T. Lee, J.W. Wang, Statistical methods for survival data analysis, 3rd ed, J. Wiley, New York, 2003.

[16] G.S. Mudholkar, D.K. Srivastava, Exponentiated weibull family for analyzing bathtub failure-rate data, IEEE Trans. Reliab. 42 (1993), 299-302.

[17] S. Nadarajah, The exponentiated exponential distribution: a survey, AStA Adv. Stat. Anal. 95 (2011), $219-251$.

[18] S. Nadarajah, S. Kotz, The beta exponential distribution, Reliab. Eng. Syst. Safety. 91 (2006), 689-697.

[19] S. Nadarajah, S. Kotz, The Exponentiated Type Distributions, Acta Appl. Math. 92 (2006), 97-111.

[20] R Core Team, R: A Language and Environment for Statistical Computing, R Foundation for Statistical Computing, Vienna, Austria, 2019.

[21] M.M. Raqab, M. Ahsanullah, Estimation of the location and scale parameters of generalized exponential distribution based on order statistics, J. Stat. Comput. Simul. 69 (2001), 109-123.

[22] M.Z. Raqab, Inferences for generalized exponential distribution based on record statistics, J. Stat. Plan. Inference. 104 (2002), 339-350.

[23] M.Z. Raqab, Generalized exponential distribution: Moments of order statistics, Statistics. 38 (2004), $29-41$.

[24] M.M. Ristić, D. Kundu, Marshall-olkin generalized exponential distribution, Metron. 73 (2015), 317-333.

[25] M.H. Tahir, S. Nadarajah, Parameter induction in continuous univariate distributions: Well-established g families, An. Acad. Bras. Ciênc. 87 (2015), 539-568. 